\title{
Genetic Logic Gates Enable Patterning of Amyloid Nanofibers
}

\author{
Ebuzer Kalyoncu, Recep Erdem Ahan, Cemile Elif Ozcelik, and Urartu Ozgur Safak Seker*
}

Distinct spatial patterning of naturally produced materials is observed in many cellular structures and even among communities of microorganisms. Reoccurrence of spatially organized materials in all branches of life is clear proof that organization is beneficial for survival. Indeed, organisms can trick the evolutionary process by using organized materials in ways that can help the organism to avoid unexpected conditions. To expand the toolbox for synthesizing patterned living materials, Boolean type "AND" and "OR" control of curli fibers expression is demonstrated using recombinases. Logic gates are designed to activate the production of curli fibers. The gates can be used to record the presence of input molecules and give output as CsgA expression. Two different curli fibers (CsgA and CsgA-His-tag) production are then selectively activated to explore distribution of monomers upon coexpression. To keep track of the composition of fibers, CsgA-His-tag proteins are labeled with nickel-nitrilotriacetic acid (Ni-NTA-) conjugated gold nanoparticles. It is observed that an organized living material can be obtained upon inducing the coexpression of different CsgA fibers. It is foreseen that living materials with user-defined curli composition hold great potential for the development of living materials for many biomedical applications.

which is attached to the cell membrane. ${ }^{[3]}$ The fibrils generally exhibit a "cross-beta spine" morphology with rows of parallel $\beta$ sheets that run perpendicular to the fiber axis which provide them good chemical and mechanical stabilities. ${ }^{[4]}$ Since modifications on CsgA monomer unit is more straightforward compared to other biofilm components such as polysaccharides, CsgA protein is used to decorate biofilms of E. coli with different proteins, peptides, and inorganic materials for building novel functional living materials. ${ }^{[5]}$

Current expression strategies of CsgA monomers in engineered circuits involve continuous demand for input molecules which limit growth of living materials. To achieve steady production of CsgA monomer upon exposure to defined input cues, we aimed to develop recombinase-based genetic logic gates as previously demonstrated. ${ }^{[6]}$ Recombinases are enzymes that are able to catalyze irreversible DNA rearrangement such as integration, inversion, and excision upon binding their recognition sites called attP and

Curli fibrils are 6-12 $\mathrm{nm}$ aggregates composed of a proteinaceous material produced and secreted to the cell surface by Enterobacteriaceae. ${ }^{[1]}$ Curli fibers are important for bacterial surface adhesion, intraspecific communication, and host colonization. ${ }^{[2]}$ Escherichia coli (E. coli) produces curli nanofibers during the biofilm formation. The main structural components of the curli fibers are the CsgA and CsgB proteins. CsgA is the major subunit and polymerizes on the CsgB protein,

Dr. E. Kalyoncu, R. E. Ahan, C. E. Ozcelik, Prof. U. O. S. Seker

UNAM - National Nanotechnology Research Center

Bilkent University

Ankara 06800, Turkey

E-mail: urartu@bilkent.edu.tr

Dr. E. Kalyoncu, R. E. Ahan, C. E. Ozcelik, Prof. U. O. S. Seker

Institute of Materials Science and Nanotechnology

Bilkent University

Ankara 06800, Turkey

Prof. U. O. S. Seker

Interdisciplinary Neuroscience Program

Bilkent University

Ankara 06800, Turkey

The ORCID identification number(s) for the author(s) of this article can be found under https://doi.org/10.1002/adma.201902888.

DOI: 10.1002/adma.201902888
attB. ${ }^{[7]}$ First, genetic circuits for Boolean type "AND" and "OR" logic control of CsgA-His tag expression using recombinases was constructed, and then the system was used to selectively activate the production of two different curli fibers (CsgA and CsgA-His-tag) to investigate the distribution of different CsgA monomers.

We obtained the two orthogonal recombinases, namely, TP901 and Bxb1 from Bonnet et al. ${ }^{[8]}$ and we constructed the dual recombinase plasmid in which the expressions of Bxb1 and TP901 are controlled by pTetO and pLacO promoters, respectively. We exploited the irreversible activity of recombinase enzymes to invert our registry DNA elements in gate plasmids to control the production of curli fibers as previously demonstrated in literature. E. coli MG1655 PRO $\Delta c s g A$ OmpR234 strain that can express LacI and TetR constitutively was used to tightly control the recombinase expression. Moreover, csgA gene knock-out enables us to eliminate any background curli production from genome yet the strain overexpresses all accessory proteins required for curli secretion owing to OmpR234 mutation. We also maintained the steady expression of CsgA monomer by using constitutive proD promoter.

To construct the "AND" gate plasmid, inversed proD promoter was placed between antialigned attP and attB recognition sites of Bxb1 while inversed his tagged CsgA coding 
(CsgA-H) with ribosome binding site and transcription terminator (rbs-CsgAH-ter) was put between antialigned attP and attB recognition sites of TP901 as depicted in Figure 1A. The dual recombinase and "AND" gate CsgAH plasmids were cotransformed in cells. After cotransformation, single colony was inoculated into LB medium with appropriate antibiotics. Next day, cells were diluted with 1:100 M63 medium supplemented with $0.2 \% \mathrm{w} / \mathrm{v}$ glucose and antibiotics. aTc and IPTG were used for the induction of recombinase enzymes TP901 and Bxb1, respectively. Cells were grown with either absence of any inducers, or in the presence of only IPTG, only aTc, and both inducers for 6 days at $30{ }^{\circ} \mathrm{C}$ without shaking. Congo red $(\mathrm{CR})$ staining was performed to verify that CsgA-His was able to form amyloid fibrils as previously described. ${ }^{[9]}$ Briefly, cells were resuspended in $25 \mu \mathrm{g} \mathrm{mL} \mathrm{m}^{-1} \mathrm{CR}$ solution, and incubated for $30 \mathrm{~min}$. Following to incubation, CR dye bound to amyloid fibrils which depleted free dissolved dye in the solution. Then, cells and CR-bound amyloid fibrils which interacted with cells through CsgB were centrifuged down to the pellet. Unbound CR remained in supernatant. Amount of unbound CR was quantified via measuring supernatant absorption at $480 \mathrm{~nm}$. $\mathrm{OD}_{600}$ of cells was measured for the normalization of $\mathrm{CR}$ binding to curli fibers. In ON state shown in truth table (Figure 1B), cells induced with both inducers showed effective binding to CR compared to only IPTG, only aTc, and uninduced cells (Figure 1C). Furthermore, we analyzed E. coli cells under scanning electron microscopy (SEM) to see fibers visually. Cells were fixed with $2.0 \%$ glutaraldehyde for overnight, and then washed with pure water and ethanol solution with increasing concentrations (25\%,50\%, 75\%, and 100\%). Prior to visualization under SEM, samples were dried with critical point dryer (CPD). No fibers were observed on uninduced cells however only aTc and only IPTG induced cells had negligible curli fiber expression compared to cells induced with both inducers. Cell clusters having dense curli fibers were present following induction with both aTc and IPTG (Figure 1D). The developed "AND" gate was functioning properly to control expression of curli production.

In the next step, we built the OR gate logic operation for curli production using the AND gate plasmid as template (Figure 2A). First, two inverted proD promoters were added between antialigned attP and attB sites of Bxb1 and TP901. Rbs-CsgAH-ter was cloned at the downstream of inverted proD promoters (Figure S3, Supporting Information). The constructed plasmid was cotransformed with dual recombinase plasmid and curli production was characterized as described above. Based on the CR staining results, cells produced curli fibers in the presence of only IPTG and only aTc; also, addition of both inducers increased the amount of CR dye binding by cells (Figure 2B). CsgA fibers were observed clearly under environmental scanning electron microscopy (ESEM) images for cells induced with only IPTG, only aTc, and IPTG-aTc. CR binding results and ESEM examination indicated that the "OR" operation circuit for curli production was working.

Recombinase enzymes invert $90 \%$ of the DNA from recognition sites in $16 \mathrm{~h}$ in vitro, ${ }^{[10]}$ therefore cells can start producing curli protein in the second day of incubation. After 3 days of incubation, media of cells were refreshed to improve biofilm formation by shutting down the unnecessary recombinase production. Since curli fibers can be disrupted or coagulated during the preparation, samples for SEM imaging of curli fibers required a careful preparation. Besides, cells and curli fibers may lose their morphologies during fixation steps. In order to minimize this, samples were dried with a critical point dryer. Curli fibers were observed as condensed and spider web-like networks in SEM images for both AND gate and OR gate controlled genetic circuits. We characterized the recombinase-based AND and the OR logic gates that control the expression of a histidine tagged CsgA protein for curli production in E. coli. These systems can be used as a starting point to build complex circuits that controls the patterning of biofilm.

Recently, Cui et al. demonstrated that addition of different polypeptides to either C- or N-terminal of CsgA changed assembly rate and length of mutant fibers. ${ }^{[11]}$ Therefore, we hypothesized that coexpression of different CsgA variants may lead to partial patterning of fibers due to different growth rate of the monomers. To do so, we took a similar approach to selectively activate the production of two different curli fibers (CsgA and CsgA-His-tag). We generated the curli patterning circuit using the OR gate plasmid as a template (Figure 3A). We cloned the rbs-csgA-terminator part into the OR gate plasmid between inverted proD promoters (Figure S4, Supporting Information). Different curli fibers were under the control of independent proD promoters. Promoters were designed with inverted orientation with respect to downstream CsgA and CsgA-His-tag (Figure 3A). Flipping of either promoter with IPTG (TP901) or aTc (Bxb1) input results in a CsgA output or CsgA-His-tag fiber, respectively. Inversion of both promoters by IPTG and aTc inputs result in a mixture of CsgA and CsgA-Histag fibers. We then transformed curli patterning circuit with dual recombinase plasmid into E. coli MG1655 PRO $\triangle \operatorname{csg} A$, and induced the cells same as described above. We then continued with CR staining to verify that CsgA and CsgA-His were able to form amyloid fibrils. CR binding to cells following the induction with only aTc and only IPTG increased compared to uninduced cells as expected. Meanwhile, addition of aTc and IPTG increased the CR binding to cells even more compared to cells induced with single agent. Coexpression of CsgA and CsgA-His monomers have no effect on curli fiber formation. To further validate this, ESEM was performed with samples from each group. Indeed, cells produced fibers subsequent to inductions and morphology of CsgA/CsgA-His mixed fibers were similar to wild-type (Figure $3 \mathrm{~B}$ ).

To visualize the composition of mixed CsgA-CsgA His fibers, we labeled fibers with Ni-NTA conjugated gold nanoparticles that have around $5 \mathrm{~nm}$ diameter. Briefly, cells were induced and collected same as described above. Resuspended cells in $1 \times$ PBS were soaked onto carbon coated transmission electron microscopy (TEM) grids. The grid was washed with deionized water and blocked with selective binding buffer $\left(1 \times\right.$ PBS, $300 \times 10^{-3} \mathrm{M}$ $\mathrm{NaCl}, 80 \times 10^{-3} \mathrm{M}$ imidazole, and $0.2 \%$ Tween-20). Then, grids were incubated onto droplet of $500 \times 10^{-9} \mathrm{M} \mathrm{Ni-NTA}$ gold nanoparticles diluted with selective binding buffer. Following to the labeling, grids were washed with selective binding buffer, $1 \times$ PBS, and deionized water, respectively. The uranium acetate staining was performed to increase the contrast, and cells were visualized under TEM. 
A)

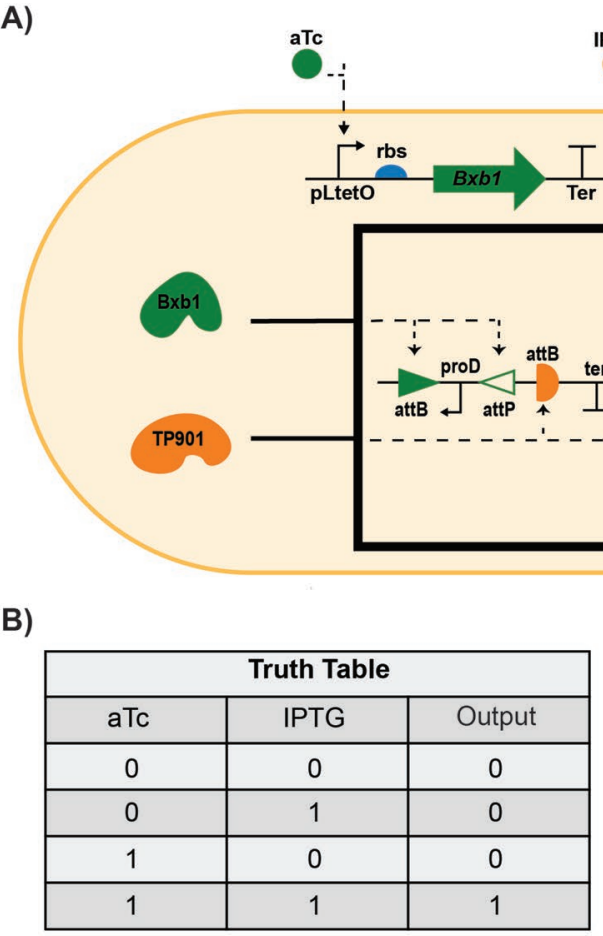

C)

Flipped by Bxb1 Flipped by TP901
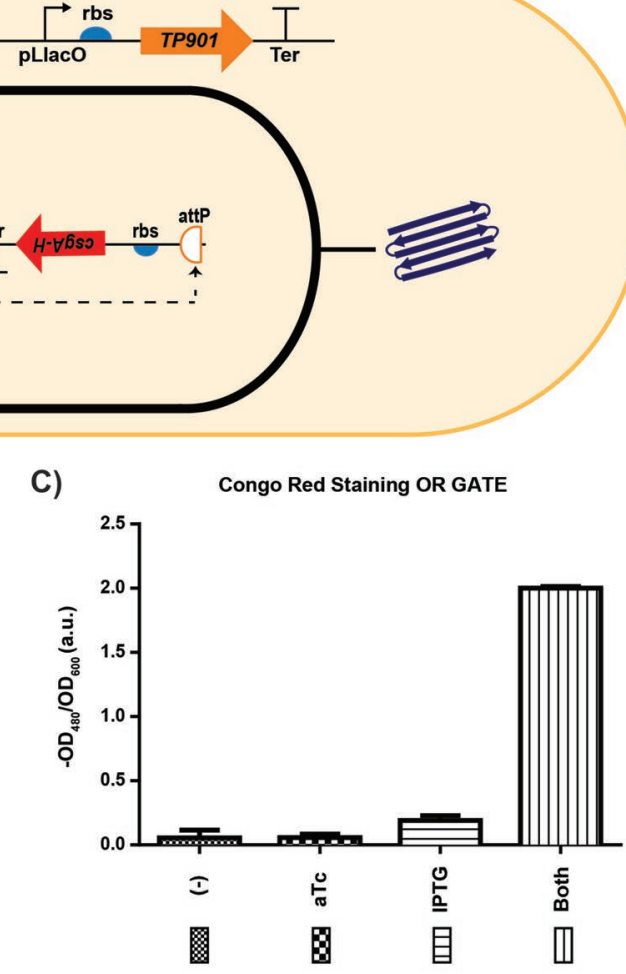

D)
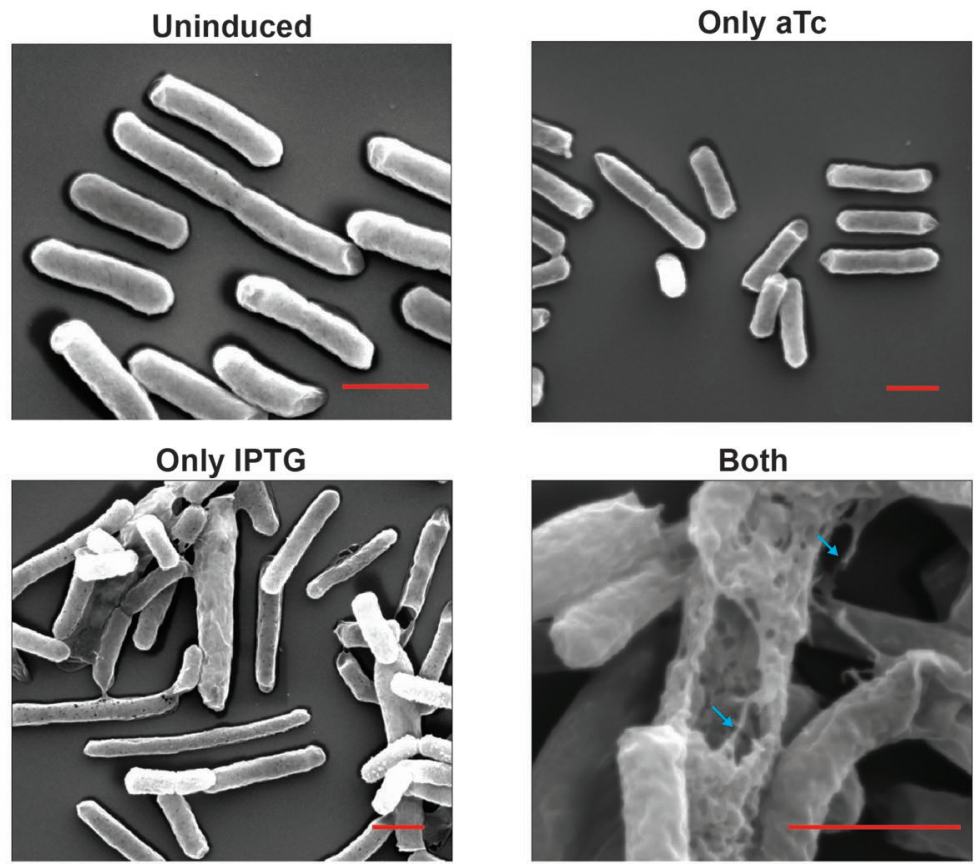

Figure 1. Production of engineered curli fibers controlled by the AND gate. A) Dual recombinase controller is with pLtetO-Bxb1 and pLlacO-TP901. Bxb1 recombinase is controlled via aTc and TP901 recombinase is controlled via IPTC. Biofilm AND logic operation system (inner circuit) is with inverted proD promoter and inverted rbs-csgA-His-tag-terminator. Inverted proD is flipped by Bxbl and inverted rbs-csgA-His-tag-terminator is flipped by TP901. B) Truth table of constructed "AND" genetic circuit. Output "1" means expression of CsgA gene which only occurs when both aTc and IPTC are added in the same time. C) Quantitative CR binding assay of cells expressing CsgA-His-tag (E. coli $\Delta$ csgA/AND logic gate) shows that CsgA-His-tag produced in the presence of both inducers. Each experiment was performed in triplicate and standard errors are shown. D) SEM images of cells that are uninduced, only aTc induced, only IPTG induced, and induced with both inducers. All scale bars are $1 \mu \mathrm{m}$. Fibers are indicated with cyan arrows. 
A)

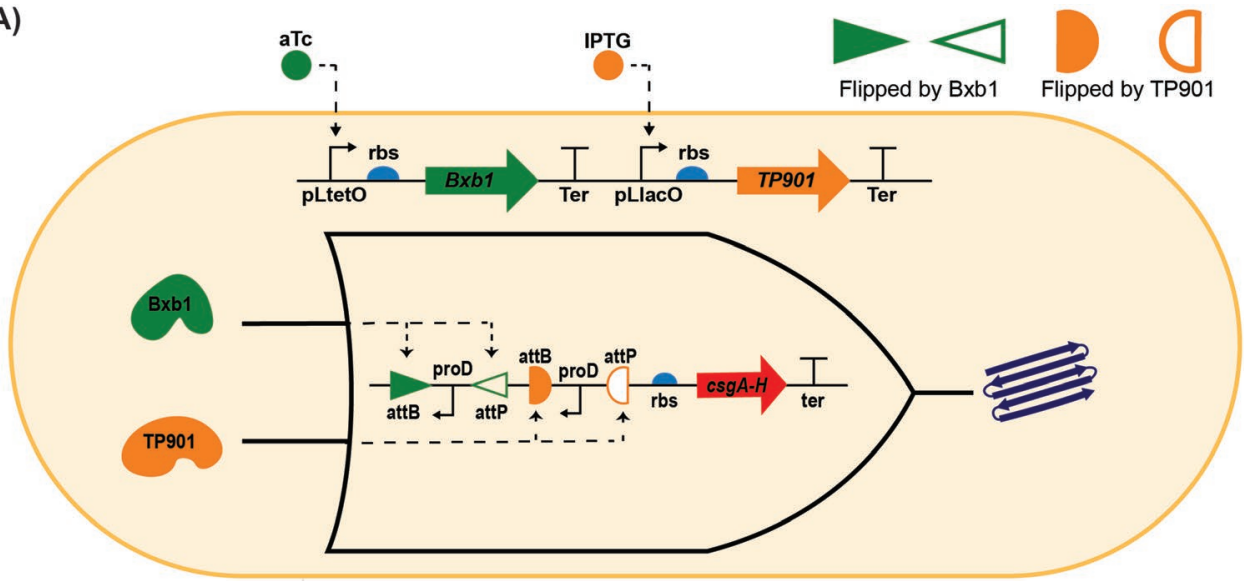

B)

\begin{tabular}{|c|c|c|}
\hline \multicolumn{3}{|c|}{ Truth Table } \\
\hline aTc & IPTG & Output \\
\hline 0 & 0 & 0 \\
\hline 0 & 1 & 1 \\
\hline 1 & 0 & 1 \\
\hline 1 & 1 & 1 \\
\hline
\end{tabular}

D)

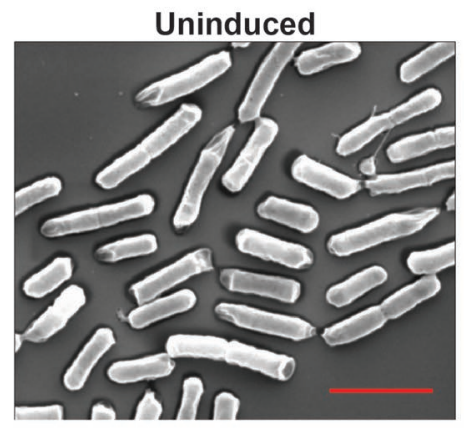

Only IPTG

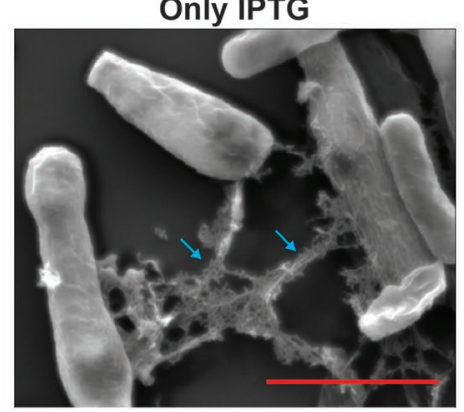

C)

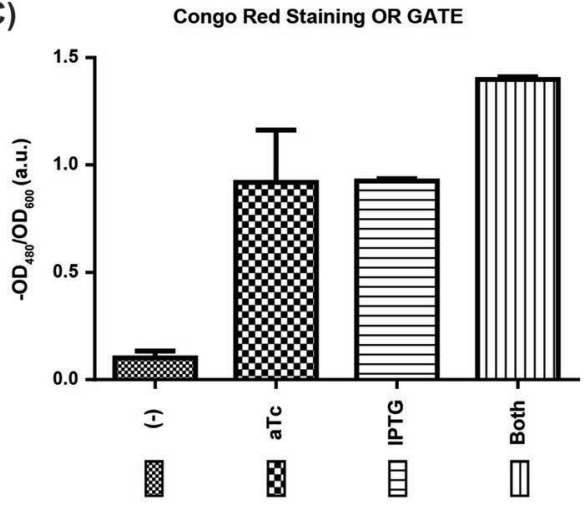

Only aTc

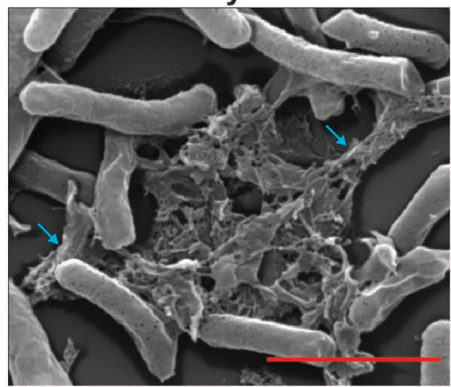

Both

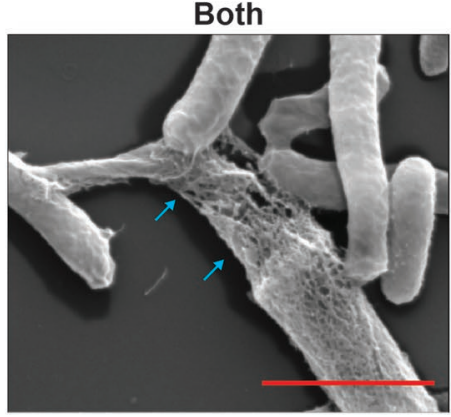

Figure 2. Production of engineered curli fibers controlled by the OR gate. A) Dual recombinase controller is with pLtetO-Bxb1 and pLlacO-TP901. Bxb1 recombinase is controlled via aTc and TP901 recombinase is controlled via IPTG. Biofilm OR logic operation system (inner circuit) is with two inverted proD promoter and rbs-csgA-His-tag-terminator. First inverted proD promoter is flipped by Bxbl and second inverted prod is flipped by TP901. Expression of CsgA-His-tag is controlled by inverted promoters. B) Truth table of constructed "OR" genetic circuit. Output "1" means expression of CsgA gene which only occur when any of the inducers are added. C) Quantitative CR binding assay of cells expressing CsgA-His-tag (E. coli $\Delta \operatorname{csg} A / O R$ logic gate) shows that CsgA-His-tag produced in the presence of any inducer. Each experiment was performed in triplicate and standard errors are shown. D) SEM images of cells that are uninduced, only aTc induced, only IPTG induced, and induced with both inducers. All scale bars are $1 \mu$ m. Fibers are indicated with cyan arrows. 
A)

B)

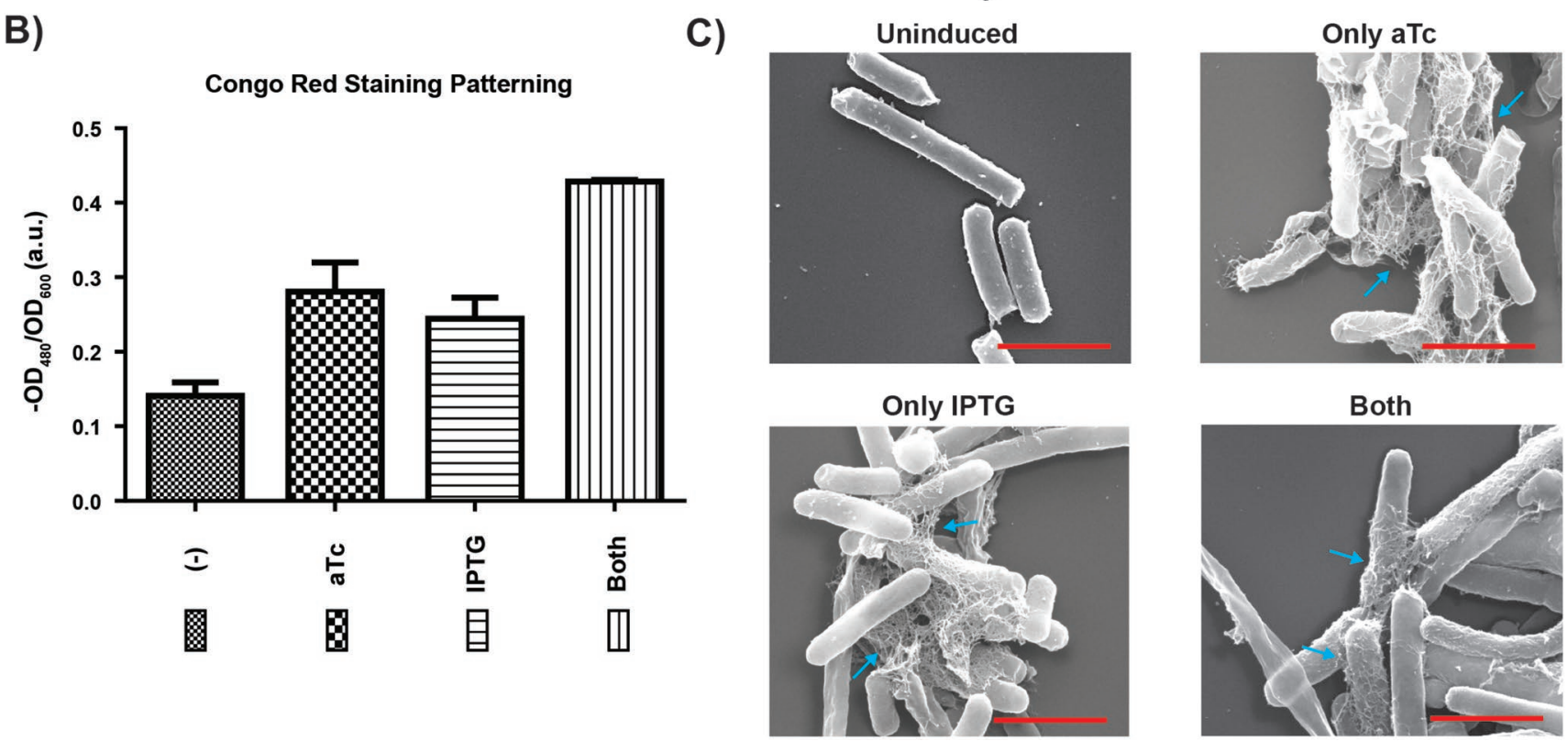

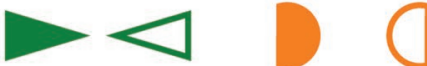

Flipped by Bxb1 Flipped by TP901

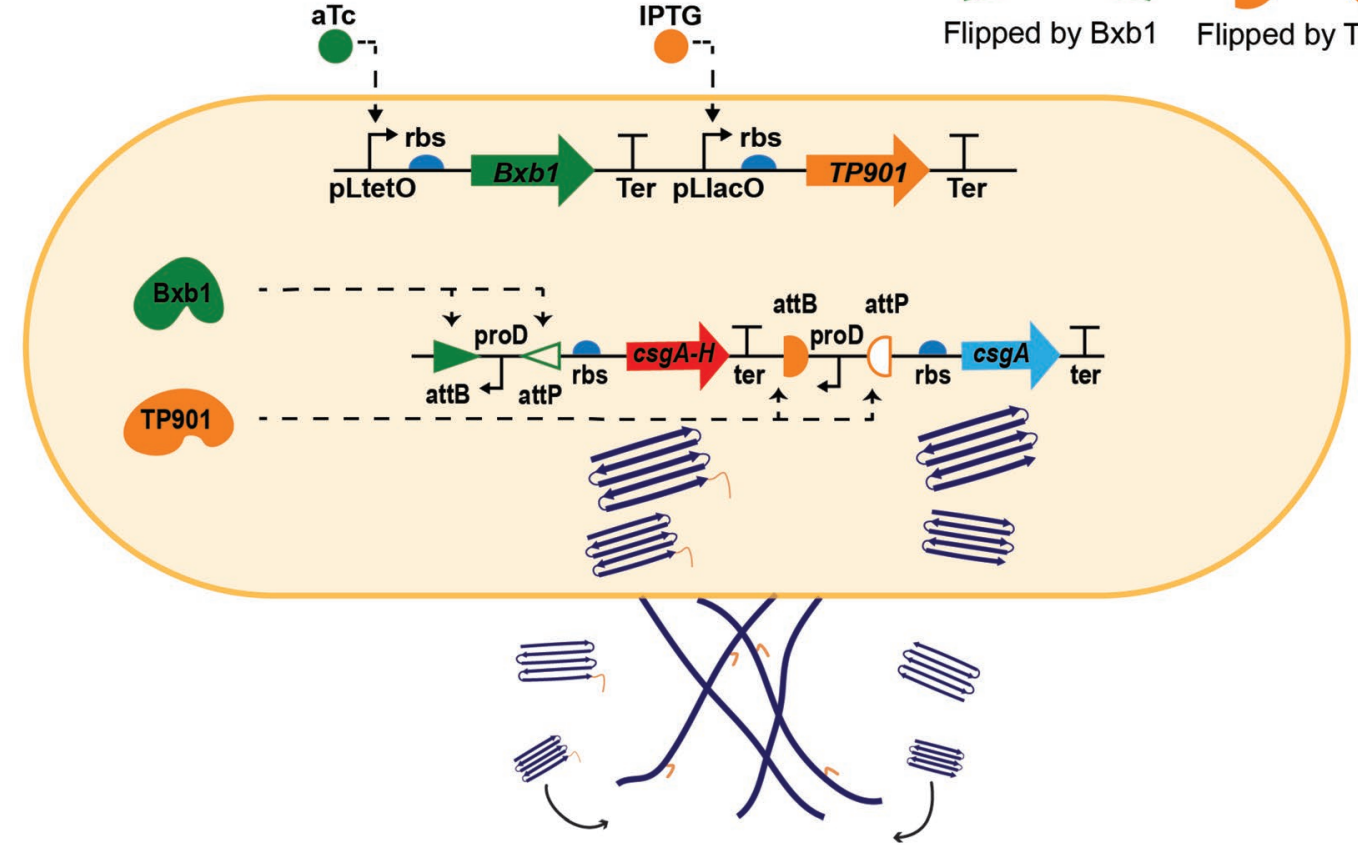

Figure 3. Production of engineered curli fibers by curli patterning gate. A) Dual recombinase controller is with pLtetO-Bxb1 and pLlacO-TP901. Bxb1 recombinase is controlled via aTc and TP901 recombinase is controlled via IPTG. Biofilm control gate (inner circuit) for patterning of curli fibers (CsgA-His, CsgA) is with one inverted proD promoter upstream of rbs-csgA-His-tag-terminator and with one inverted proD promoter upstream of rbs-csgA-terminator. First inverted proD promoter is flipped by Bxb1 and second inverted prod is flipped by TP901. CsgA-His expression occurs when aTc is present and csgA expression occurs when IPTC is present. B) Quantitative CR binding assay of cells expressing CsgA-His-tag and CsgA (E. coli $\Delta c s g A / C u r l i$ patterning gate) shows that CsgA-His-tag produced in the presence of aTc and CsgA produced in the presence of IPTC. Each experiment was performed in triplicate and standard errors are shown. C) SEM images of cells that are uninduced, only aTc induced, only IPTC induced, and induced with both inducers. All scale bars are $1 \mu \mathrm{m}$. Fibers are indicated with cyan arrows.

TEM images revealed that both CsgA and CsgA-His fibers shares similar morphology as seen in ESEM previously. CsgAHis fibers were selectively labeled with gold nanoparticles in the presence of relatively high imidazole concentration. For coexpression of CsgA and CsgA-His fibers continuously for
6 days, labeled fibers accumulated in certain regions rather than scattered randomly among the other fibers (Figure 4). CsgA-His and CsgA fibers segregated from each other at certain degree in different fibers when they were coexpressed for long times. 
Uninduced
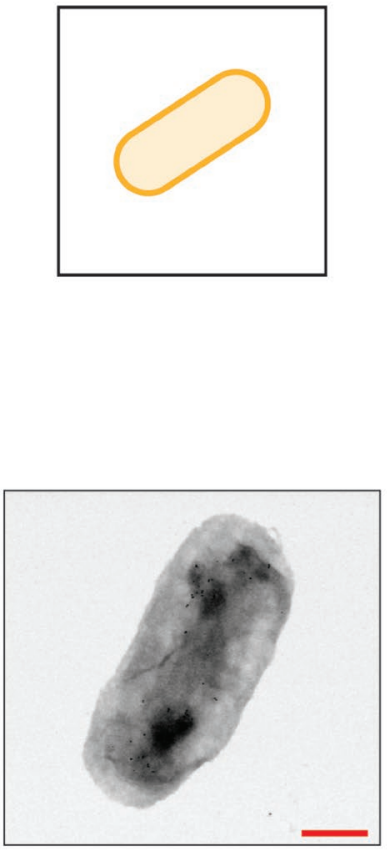

Only aTc
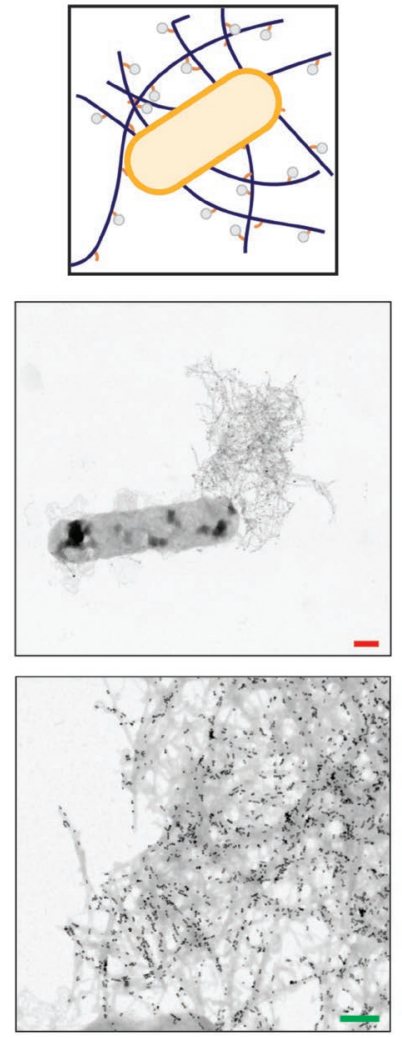

Only IPTG
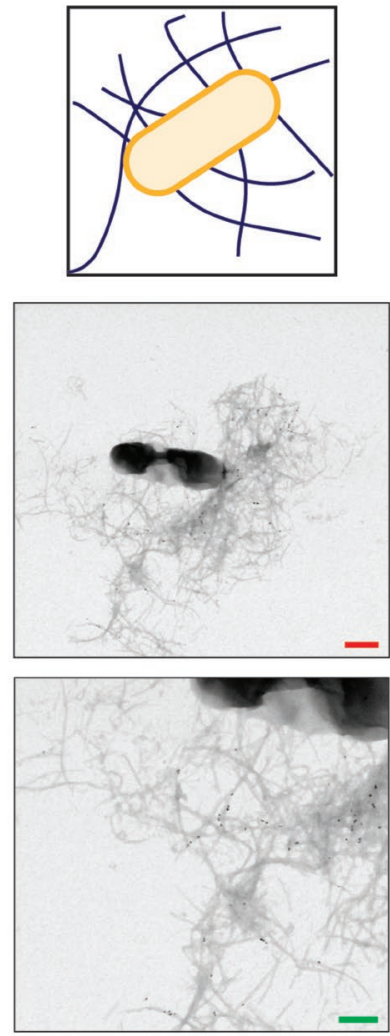

Both
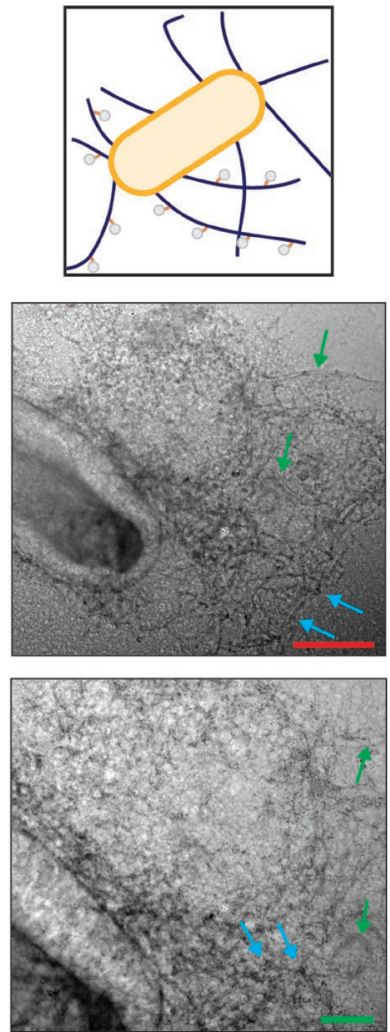

Figure 4. Curli patterning with Ni-NTA-AuNPs labels via recombinase-based regulation with inducers. E. coli $\triangle \operatorname{csg} A$ cells that are carrying curli patterning gate plasmid were used to form amyloid fibrils composed of CsgA-His and/or CsgA in the presence of aTc and/or IPTC. CsgA-His was labeled by Ni-NTA conjugated AuNPs and visualized under TEM. The down panel is the zoom-in view of upper panel for the only aTc, only IPTG, and aTc-IPTC induced cells. Labeling of cells that are expressing CsgA-His-tag and CsgA (E. coli $\Delta$ csgA/Curli patterning gate) with AuNPs shows that CsgAHis-tag produced in the presence of aTc, CsgA produced in the presence of IPTG, and fiber mixtures that are composed of CsgA-His-tag and CsgA produced in the presence of both inducers. Expression of both CsgA and CsgA-His monomers at the time leads to partial segregation of monomers in different fibrils. Green arrow indicates the fibrils that are high CsgA monomer while cyan arrow indicates fibrils that have high content of CsgA-His variant. Red and green scale bars are 500 and $200 \mathrm{~nm}$.

In summary, we show that recombinase-based genetic logic gates can be engineered to control production of functional curli fibers in living cells. For all circuits, cell viabilities were similar to each other and there were at least $2.5 \times 10^{8} \mathrm{~mL}^{-1}$ alive cells in the culture (Figure S2, Supporting Information). Genetic circuits designed with two-input Boolean logic functions were shown in many studies. ${ }^{[6,12]}$ Logic operations enable specific activation of genetic circuits when defined conditions are met. By using recombinases, input molecules are recorded as CsgA production. Even if input signals are gone, CsgA monomers continue to be expressed from cell until the loss of the plasmid that encodes CsgA coding region and promoter. In a recent study, we demonstrated that biofilm formation through overexpression of $\operatorname{csg} A$ gene increases stability of cells in catalysis action at high temperature and in prolonged growth. ${ }^{[13]}$ The developed strategy can be used to induce CsgA expression upon exposure stress conditions which makes the cell catalysts more stable compared to planktonic cells; moreover, readout of stress exposure would be recorded as biofilm formation. Another possible application of the proposed system can be bioremediation of heavy metal contaminated soils. Biosensing of contaminant ions such mercury, cadmium, or arsenic is possible through responsive promoters ${ }^{[14]}$ and corresponding metal immobilizing CsgA variants can be expressed upon sensing. A simple version of CsgA-based bioremediation material is demonstrated by Tay et al. ${ }^{[15]}$

Natural cues can be utilized to adjust the extracellular matrix properties of biofilms which pave the way for designing elegant materials. In our previous study, we showed that mechanical properties can be tuned by changing the composition of curli fibers using different CsgA variants. ${ }^{[16]}$ Stiffness of materials that are used in stem cell culturing is shown as a critical parameter in cell differentiation and self-renewal. ${ }^{[17]}$ Living base materials that evolve their material properties based on the cell status can be engineered for organoid formation and stem cell engineering. ${ }^{[18]}$ In addition, patterning of E. coli cells on textile materials such as cotton and plastics through functionalized CsgA variants makes it possible to develop responsive clothes that can sense small molecules or replace the body odor with more pleasant scents. ${ }^{[19]}$ Besides, novel devices such as dome-shaped pressure sensors can be built via controlling the CsgA expression in cells with 3D patterning circuit. ${ }^{[20]}$ As an 
electronic conduit between electronic devices and cells, conductive biofilms that carry modified CsgA monomers were shown in several studies. ${ }^{[21]}$ Responsive and multifunctional biomaterial fabrication is a growing and demanding topic that can provide innovative solutions to advance areas such as healthcare and electronics when combined with synthetic biology approaches. Therefore, recent developments in engineered living materials were reviewed by Gilbert and Ellis. ${ }^{[22]}$

In the present study, we developed a recombinase-based approach as a new tool that can be used to produce CsgA variants with different functions, enabling "smart" material fabrication. From our perspective, living materials that can respond and change their characteristic properties such as elasticity, stiffness, conductivity, and composition will endow new approaches for future applications.

\section{Supporting Information}

Supporting Information is available from the Wiley Online Library or from the author.

\section{Acknowledgements}

The study was supported by TUBITAK Grant Numbers $114 \mathrm{M} 163$ and 216M127. U.O.S.S. thanks the TUBA-GEBIP Award. E.K. thanks TUBITAK BIDEB Graduate Fellowship.

\section{Conflict of Interest}

The authors declare no conflict of interest.

\section{Keywords}

amyloid fibers, biofilm patterning, genetic circuits, genetic logic gates, recombinase

Received: May 6, 2019

Revised: July 15, 2019

Published online: August 12, 2019

[1] M. R. Chapman, L. S. Robinson, J. S. Pinkner, R. Roth, J. Heuser, M. Hammar, S. Normark, S. J. Hultgren, Science 2002, 295, 851.

[2] a) X. Zogaj, M. Nimtz, M. Rohde, W. Bokranz, U. Romling, Mol. Microbiol. 2001, 39, 1452; b) U. Gophna, T. A. Oelschlaeger, J. Hacker, E. Z. Ron, FEMS Microbiol. Lett. 2002, 212, 55; c) U. Gophna, M. Barlev, R. Seijffers, T. A. Oelschlager, J. Hacker, E. Z. Ron, Infect. Immun. 2001, 69, 2659; d) J. W. Austin, G. Sanders, W. W. Kay, S. K. Collinson, FEMS Microbiol. Lett. 1998, 162, 295; e) X. Zogaj, W. Bokranz, M. Nimtz, U. Romling, Infect. Immun. 2003, 71, 4151 .

[3] M. M. Barnhart, M. R. Chapman, Annu. Rev. Microbiol. 2006, 60, 131.

[4] a) E. P. DeBenedictis, D. Ma, S. Keten, RSC Adv. 2017, 7, 48102; b) R. Nelson, M. R. Sawaya, M. Balbirnie, A. O. Madsen, C. Riekel, R. Grothe, D. Eisenberg, Nature 2005, 435, 773; c) M. Sunde, L. C. Serpell, M. Bartlam, P. E. Fraser, M. B. Pepys, C. C. F. Blake, J. Mol. Biol. 1997, 273, 729; d) P. F. Tian, W. Boomsma, Y. Wang, D. E. Otzen, M. H. Jensen, K. Lindorff-Larsen, J. Am. Chem. Soc. 2015, 137, 22

[5] a) P. Q. Nguyen, Z. Botyanszki, P. K. R. Tay, N. S. Joshi, Nat. Commun. 2014, 5, 4945; b) A. Y. Chen, Z. Deng, A. N. Billings, U. O. Seker, M. Y. Lu, R. J. Citorik, B. Zakeri, T. K. Lu, Nat. Mater. 2014, 13, 515; c) U. O. S. Seker, A. Y. Chen, R. J. Citorik, T. K. Lu, ACS Synth. Biol. 2017, 6, 266.

[6] P. Siuti, J. Yazbek, T. K. Lu, Nat. Biotechnol. 2013, 31, 448.

[7] C. A. Merrick, J. Zhao, S. J. Rosser, ACS Synth. Biol. 2018, 7, 299.

[8] J. Bonnet, P. Yin, M. E. Ortiz, P. Subsoontorn, D. Endy, Science 2013, 340, 599.

[9] a) M. R. Nilsson, Methods 2004, 34, 151; b) C. Reichhardt, A. N. Jacobson, M. C. Maher, J. Uang, O. A. McCrate, M. Eckart, L. Cegelski, PLoS One 2015, 10, e0140388.

[10] S. M. Stoll, D. S. Ginsburg, M. P. Calos, J. Bacteriol. 2002, 184, 3657.

[11] M. Cui, Q. Qi, T. Gurry, T. Zhao, B. An, J. Pu, X. Gui, A. A. Cheng, S. Zhang, D. Xun, Chem. Sci. 2019, 10, 4004.

[12] B. J. Wang, R. I. Kitney, N. Joly, M. Buck, Nature 2011, 480, E2.

[13] R. E. Ahan, B. Saltepe, O. Apaydin, U. O. S. Seker, ChemBioChem 2019, 20, 1799.

[14] B. Saltepe, E. S. Kehribar, S. S. Su Yirmibeşoğlu, U. O. S. Şeker, ACS Sens. 2018, 3, 13.

[15] P. K. R. Tay, P. Q. Nguyen, N. S. Joshi, ACS Synth. Biol. 2017, 6, 1841.

[16] M. T. Abdelwahab, E. Kalyoncu, T. Onur, M. Z. Baykara, U. O. S. Seker, Langmuir 2017, 33, 4337.

[17] a) N. Gjorevski, N. Sachs, A. Manfrin, S. Giger, M. E. Bragina, P. Ordóñez-Morán, H. Clevers, M. P. Lutolf, Nature 2016, 539, 560; b) N. Broguiere, L. Isenmann, C. Hirt, T. Ringel, S. Placzek, E. Cavalli, F. Ringnalda, L. Villiger, R. Züllig, R. Lehmann, Adv. Mater. 2018, 30, 1801621.

[18] J. J. Hay, A. Rodrigo-Navarro, M. Petaroudi, A. V. Bryksin, A. J. García, T. H. Barker, M. J. Dalby, M. Salmeron-Sanchez, Adv. Mater. 2018, 30, 1804310.

[19] a) F. Moser, E. Tham, L. M. González, T. K. Lu, C. A. Voigt, Adv. Funct. Mater. 2019, 29, 1901788; b) X. Liu, T.-C. Tang, E. Tham, H. Yuk, S. Lin, T. K. Lu, X. Zhao, Proc. Natl. Acad. Sci. USA 2017, 114, 2200; c) J. Dixon, N. Kuldell, in Methods in Enzymology, Vol. 497 (Ed: C. Voigt), Elsevier, New York 2011, p. 255.

[20] Y. X. L. Cao, Y. Y. Feng, M. D. Ryser, K. Zhu, G. Herschlag, C. Y. Cao, K. Marusak, S. Zauscher, L. C. You, Nat. Biotechnol. 2017, 35, 1087.

[21] a) E. Kalyoncu, R. E. Ahan, T. T. Olmez, U. O. S. Seker, RSC Adv. 2017, 7, 32543; b) N.-M. D. Courchesne, E. P. DeBenedictis, J. Tresback, J. J. Kim, A. Duraj-Thatte, D. Zanuy, S. Keten, N. S. Joshi, Nanotechnology 2018, 29, 454002.

[22] C. Gilbert, T. Ellis, ACS Synth. Biol. 2019, 8, 1. 